\title{
Chronic Orofacial Pain, Central Sensitization and Sleep - Is There a Link?
}

\section{Francisco Alencar Jr*}

General Dental Sciences Department, TMD and Orofacial Pain, Marquette University School of Dentistry, Milwaukee, WI, USA

Pain has been cited in very ancient texts such as the Old and New Testament in the Bible. However, it was only defined in the scientific literature in 1986 when the International Association for the Study of Pain (IASP) provided officially their first classification and pain description [1].

Contrary to what one might think, pain cannot be defined only as an unpleasant sensation. Pain is a highly subjective experience that involves the peripheral and central nervous system and is influenced by previous experiences, emotional and cognitive factors. It has long been appreciated that the experience of pain is highly variable between individuals. Pain results from activation of sensory receptors specialized to detect actual or impending tissue damage (nociceptors). Nevertheless, a direct correlation between activation of nociceptors and the sensory experience of pain is not always apparent. Even in cases in which the severity of injury appears similar, individual pain experiences may vary dramatically. Emotional state, degree of anxiety, attention and distraction, past experiences, memories, and many other factorscan either enhance or diminish the pain experience. This is what we call pain modulation [2].

Pain could also be viewed as a protective or defensive mechanism that sends the brain an alert so a protective behavior can be generated. Nevertheless in Chronic Pain this purpose is missing. When pain lasts longer than it is supposed after healing has happened, it can in fact turn to be an etiologic factor. Therefore pain cannot be viewed only as a sign or a symptom of a disease. This finding has a profound impact in the way Chronic Pain should be viewed by health care providers. Also it should create a shift in patient's behavior towards pain management. In other words, this means that treating pain is not only palliative or supportive care. When managing pain properly we are in fact treating or controlling a potential or actual etiologic factor. Pain can cause transient or irreversible changes in the peripheral and central nervous system, clinically resulting in Myofascial Pain, Neuropathic Pain and other complex pain syndromes.

There has been an extensive debate towards the length or duration of pain to be considered chronic. Usually 3 months or 6 months would be considered the minimal pain duration. The question is: Why is it important to differentiate between chronic and acute pain? The importance relies in the need to treat these patients with a different approach. The difference is usually related to the presence of Central Sensitization, Sleep Dysfunction and severe Psychological Issues. Therefore the debate about time/duration becomes of a secondary importance since the presence of signs or symptoms of Central Sensitization and Poor Sleep quality becomes the key topics in Chronic Pain definition and consequently management.

In order to understand Central Sensitization we need to consider pain not as a sign or symptom of a disease or pathological disorder. We need to see pain with a potential to cause damage to the Central Nervous System (CNS) according to its severity, intensity and duration. In other words, the longer the duration and the greater the pain intensity, chances are that some sort of damage will occur in the CNS. Central sensitization represents an enhancement in the function of neurons and circuits in nociceptive pathways caused by increases in membrane excitability and synaptic efficacy. The whole CNS becomes oversensitive and will respond not only to painful stimulations, but rather to all sorts of excitations in a very different and peculiar way. This change may affect different autonomic, sensory and motor fibers.

When affecting autonomic fibers the patient can clinically manifest signs and symptoms that will resemble an allergic or sinus infection. These could be facial swelling, increase in skin temperature, redness, eye lacrimation, rhinorrhea, nasal congestion, sneeze and cough among others. Sensory or sensitive fibers may be affected and present clinically as allodynea or secondary hyperalgesia. Motor fibers can initially present muscle protective co-contraction that if postergated will originate muscle myalgia and perhaps myofascial pain. Interesting to note is that all these different clinical presentations are constantly misdiagnosed and they are all caused by constant pain input.

It is not surprising that patients with chronic headaches have a higher prevalence of myofascial trigger points, and headache symptoms that can be reproduced by stimulation of an active trigger point [3]. Through my research I have found that the presence of trigger points may be a contributing factor in the initiation and/or perpetuation of chronic headaches. Myofascial pain can be the cause, along with the evidence of central sensitization, of "atypical facial pain", with trigger points referring pain to the teeth, gums, maxillary sinus, mandible, ears, face, head and /or some kind of vision impairment [4]

When a referred patient presents for an emergency consult indicating tooth pain, he could actually be suffering from Myofascial Pain Disorder (MPD.) All practitioners who treat pain patients, especially chronic pain patients, need to study and better understand the etiology in order to come out with an adequate diagnosis. Considering only occlusion or psychological issues as potential etiologic factors to TMD or MPD is not considered evidence based Dentistry.

Patients have undergone invasive surgery procedures, including teeth extractions and TMJ surgery, when the diagnosis was myofascial pain or neuropathic pain. Neuropathic pain etiology used to be linked only when some nerve damage had occurred. In fact this can be one of the causes of neuropathic pain, but inadequately managed chronic pain can lead to central sensitization and neuropathic pain as well. This can be caused due to changes in the peripheral and central nervous system. It can be even more confusing because it is usually described as a "burning, electrical shock like or throbbing pain." If this pain is being felt in some teeth or head, can be easily confused and diagnosed as odontogenic pain or migraine.

Evidence based Dentistry points in one direction for the management of Chronic TMD and other Orofacial Pain conditions. It is now known

*Corresponding author: Francisco Alencar Jr. DDS, MS, MS, PhD, General Dental Sciences Department, TMD and Orofacial Pain, Marquette University School of Dentistry, Milwaukee, WI, USA, Tel: 414-288-5590; E-mail: docfalencarjr@yahoo.com, fguedes00@yahoo.com

Received November 04, 2013; Accepted November 06, 2013; Published November 08, 2013

Citation: Alencar F (2013) Chronic Orofacial Pain, Central Sensitization and Sleep- Is There a Link? Dentistry 3: e112. doi:10.4172/2161-1122.1000e112

Copyright: $\odot 2013$ Alencar F. This is an open-access article distributed under the terms of the Creative Commons Attribution License, which permits unrestricted use, distribution, and reproduction in any medium, provided the original author and source are credited. 
that a combination of many factors contribute to this condition. Sleep quality, daytime posture, habits including work environment, posture during sleep, insufficient hydration, caffeine intake, nicotine intake, daytime parafunctions and sleep bruxism, anxiety, frustration and depression, can all affect the Central Nerve System (CNS) and cause more pain or perpetuate the pain through central sensitization. Inadequate occlusion could be a consequence and not the cause of inflammation in the temporomandibular joint. In multiple cases with TMD and/or TMJ inflammation the patient completed treatment with a much better an adequate occlusal relationship.

Chronically painful conditions are frequently associated with sleep disturbances, i.e. changes in sleep continuity and sleep architecture as well as increased sleepiness during daytime. A new hypothesis, which has attracted more and more attention, is that disturbances of sleep cause or modulate acute and chronic pain. Since it is wellknown that pain disturbs sleep, the relationship between the two has since recently been seen as reciprocal. According to the majority of the studies, sleep deprivation produces hyperalgesic changes. Furthermore, sleep deprivation can interfere with analgesic treatments involving opioidergic and serotoninergic mechanisms of action [5]. This is very important information that clinicians dealing with chronic pain should never forget.

Laboratory based sleep deprivation studies have also suggested that reductions in total sleep time are accompanied by increased sensitivity to noxious stimuli (secondary hyperalgesia) and by decrements in endogenous pain-inhibitory processes. This could also be a link between chronic pain and central sensitization (CS), since secondary hyperalgesia and other secondary excitatory effects specifically related to CS could be related to a longer duration of the pain causing a reduction of the sleep time. Alternatively, a relatively long sleep period may indicate poor sleep continuity (e.g., fragmented, or overly "light" sleep), which has been associated with enhanced pain perception in some laboratory studies of experimental sleep fragmentation [6].

It is a very complex area due to the numbers of factors that can be involved, the need for the clinician to study other areas not related directly with Dentistry and the controversies in the literature most of the times caused by grouping a lot of different subtypes of TMD diagnosis in one big group and call it TMD.

It seems to be clear and evident that sleep and pain have a close relationship. Also, chronic pain can cause sleep disturbances and central sensitization. Health care providers who treat patients in pain must be familiar with these concepts as well as treatment modalities to manage them.

\section{References}

1. Mersky H (1986) Classification of chronic pain: Description of chronic pain syndromes and definition of pain terms. Pain 3: S1-226.

2. Ossipov MH, Dussor GO, Porreca F (2010) Central Modulation of Pain. J Clin Invest 120: 3779-3787.

3. Sanitá PV, Alencar Jr FGP (2009) Myofascial Pain Syndrome as a contributing factor in patients with chronic headaches. Journal of Musculoskeletal Pain 17 $15-25$

4. Alencar Jr FGP (2010) The Puzzling Problems of Myofascial Pain. Marquette University Discover Magazine.

5. Lautenbacher S, Kundermann B, Jürgen-Christian Krieg (2006) Sleep deprivation and pain perception. Sleep Medicine Reviews 10: 357-369.

6. Everson CA, Szabo A (2009) Recurrent restriction of sleep and inadequate recuperation induce both adaptive changes and pathological outcomes. Am J Physiol Regul Integr Comp Physiol 297: 1430-1440. 